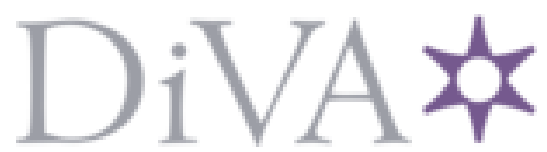

http://www.diva-portal.org

\title{
Postprint
}

This is the accepted version of a paper published in Asia Pacific Journal of Anthropology. This paper has been peer-reviewed but does not include the final publisher proof-corrections or journal pagination.

Citation for the original published paper (version of record):

Davey, G., Zhao, X. (2021)

Smoking and the City: A Travelogue in Yuxi

Asia Pacific Journal of Anthropology, 22(1): 58-80

https://doi.org/10.1080/14442213.2021.1889652

Access to the published version may require subscription.

N.B. When citing this work, cite the original published paper.

Permanent link to this version:

http://urn.kb.se/resolve?urn=urn:nbn:se:oru:diva-90695 


\title{
Accepted version
}

\section{Smoking and the City: A Travelogue in Yuxi}

\author{
Gareth Davey ${ }^{\star *}$ and Xiang Zhao
}

We narrate our travel in a city with a prominent tobacco company to contribute to the literature on smoking and place. We show that in Yuxi, the location of the Hongta Group, China's largest tobacco manufacturer, a smoker's sense of self and of place is affectively, cognitively and socially tied to the tobacco industry. Tobacco-related symbolism in the city is highly visible and abundant. Smoking and tobacco are enmeshed in the fabric of the city and of people's lives, an integrated and totalising phenomenon we characterise as 'rhizomatic smoking'. Hence, we call for 'rhizomatic tobacco control' as a new direction for conceptualising the entirety of tobacco in society and for subjecting tobacco control in China to critical scrutiny.

Keywords: Hongta Group; Yuxi; Hongtashan; Smoking; Tobacco Industry; China

\section{Introduction: Enroute to Yuxi}

Our bus was meandering through an endless sea of golden-coloured tobacco fields, a sign that we were enroute to Yuxi, a prefecture-level city in Yunnan Province, China, and the location of the Hongta ${ }^{1}$ Group-one of the largest tobacco companies in China and the world with an annual production of 93 billion cigarettes (Li 2012). The tobacco industry in Yuxi was prioritised in the late 1970s following economic and governance freedoms in cities and provinces, and about 80 per cent of the city's revenue now comes from tobacco taxes (Li 2012; Wang 2012). Yuxi has since become well known in China as the 'city of tobacco', like the industry-associated identities of many cities globally (Cheshmehzangi 2020).

Research on health and place is increasing, motivated partly by geographical inequalities in health and healthcare. Smoking, a leading cause of death and disease, is linked to numerous background and contextual factors independent of

* Gareth Davey and Xiang Zhao are affiliated with the School of Foreign Languages and Literature at Yunnan Normal University. Correspondence to: Xiang Zhao, Research Centre for Languages and Cultures, School of Foreign Languages and Literature, Yunnan Normal University, Kunming 650500, Yunnan Province, China. E-mail: rclc@tutamail.com; rclc@ynnu.edu. 
the characteristics of individuals (Barnett et al. 2017). For example, health facilities (Stead et al. 2001), tobacco retailers (Pearce et al. 2009), tobacco control (Li and Collins 2017), crime and discontent (Stead et al. 2001), urbanisation (Pearce and Boyle 2005), respite and recreation (Stead et al. 2001) and urban deprivation (Stead et al. 2001). Most studies, however, have been conducted in Western countries and fail to capture the complexity of smoking and place. Initial research in China indicated that overall smoking prevalence was highest in provinces with the highest rates of cigarette production (Yang et al. 2015), an association also shown in other countries (Morley and Pratte 2013). One reason could be economic dependence on tobacco manufacturing. Provincial governments are influential stakeholders in the tobacco industry with a vested economic interest in stimulating cigarette production and consumption (Yang et al. 2015). Tobacco advertising and promotion may be important too: a study of adult smokers in six Chinese cities found that tobacco advertisements were noticed the most in cities with a comparably greater tobacco industry presence, and the noticing of advertisements was associated with more positive attitudes towards and support for the tobacco industry (Yang et al. 2010). Other tentative explanations for the association between smoking prevalence and cigarette production include access to cigarettes through retailing practices, cultural and social practices, tobacco control, and high community profiles and lobbying practices of tobacco companies (Yang et al. 2015).

We build on the above literature by examining the significance of self and identity in smoking and place in a specific city where tobacco is manufactured, and by examining how tobacco products infiltrate people's lives. Classic studies (for example, Erikson 1950) underlined the importance of social environment in self-identity development. Key thinkers on place such as Anne Buttimer and David Seamon (1980), Edward Relph (1976) and Yi-Fu Tuan (1980) demonstrated that people develop profound existential and psychological ties through attachment to places, and recent work has highlighted the importance of identity in place and smoking (Barnett et al. 2017). In China, smoking affords a sense of belonging, meaning and purpose, and is entwined in social practices, perceptions of health and social changes (for example, Davey and Zhao 2020; Zhao and Davey 2015). Smoking and cigarettes express identities, taking on messages about class, emotion, face-making, fashion, gender, glamour, health, status, success, and wealth, for instance (Davey and Zhao 2012, 2020; Rich and Xiao 2012). People who smoke choose cigarette brands and cigarette packet designs that fit their personal perceptions of identity and to assert identity and individuality (Davey and Zhao 2020; Scheffels 2008). Smoking accessories, such as cigarette cases and lighters, have been designed to appeal to young people in China by conveying an 'ideal smoker' identity (Mak 2012). However, research on the interplay of identity, smoking and place in China is scarce.

In this travelogue we narrate our fieldtrips in Yuxi and our interviews with people who smoked, ${ }^{2}$ supplemented with photographs of the city, newspaper articles and our impressions and memories, to examine the impact of the tobacco industry on smoking and smoker identities. Before we take you on our journey, it is instructive 
to define our approach and objectives. We were particularly interested in smokingrelated meanings and practices among people who smoked in Yuxi, our first research objective. Second, we have described some of the tobacco-related symbolism we saw in the city (for example, in advertising, branding, structural forms such as architecture and settings, and smoking prevalence and visibility) and in broader society (for example, economic and political ideology), and the ways they are worked into everyday life to legitimise smoking and to ensure its continuance. We have also considered representations of the city in official Chinese newspaper content from 1946 to 2018, our third research objective, as the social construction of places in society influences the formation of their identities and the internalisation and expression of such identities by residents (Cheshmehzangi 2020). Fourth, we have pondered the implications of living within the vicinity of the Hongta Group on smoking. Thus, smoking, identity and place in Yuxi have been examined in our study from varying perspectives, from their cognitive foundations to their expression in the tangible and intangible forms in the city, part and parcel of the same phenomena-as any separation here is simply for emphasis and analysis. We offer a refreshing change from most smoking research in China by providing a qualitative and more nuanced examination of smoker identities in contrast to the usual big quantitative data studies and, also, by focusing on one city, as small scale studies on smoking and place are still a rarity.

It is also crucial to subject our own position to critical scrutiny. As reflexive researchers we recognise that the assumptions of our research are not inevitable or universal and that alternative positions exist. Gareth Davey trained in anthropology, health psychology and public health; and Xiang Zhao trained in counselling, health psychology and tobacco control. Motivated by statistics on tobacco-related morbidity and mortality in China, we have primarily adopted a public health stance in our tobacco research that is anchored on the minimisation and cessation of smokingin line with mainstream tobacco research. A recent innovation in the literature on the anthropology of smoking and of tobacco is interrogation of the polarisation of scholarship along the lines of a public health/tobacco control framework versus a tobacco industry framework, and the potential harm to scholarship entailed by either party in pursuing their interests, notably by narrowing the field of enquiry and leaving little room to understand tobacco outside a tobacco control agenda (Bell and Dennis 2013; Dennis 2016). Therefore, it is essential that we reflect throughout our essay on the positionedness of our research in terms of its commitment to ending the global tobacco epidemic, not because helping to bring about smoking cessation should be stopped but to realise that alternative positions exist that might tell us something different about the meanings of smoking and the social context in which it is practised and experienced (Dennis 2016).

\section{Our Arrival: Hongta District}

We arrived at the bus terminal in Hongta District, Yuxi's economic and political centre. Immediately apparent in this eponym was the Hongta Group's omnipresence 
in the cityscape. The company's name adorns signature buildings, roads and areas, to build public support and a positive image: Hongta Avenue, Hongta Cultural and Sports Centre, Hongta Forest Park, Hongta Hotel, Hongta Industrial Tourism Park and Hongta Sports Stadium, to name a few. We could not but feel the grandeur and greatness of the tobacco industry embodied in these places that also portray tobacco as commonplace and integral to everyday life and society-our vivid first impressions of the city.

Indeed, most of the people we met in Yuxi talked favourably about the Hongta Group. They praised its contributions to the city and province, especially revenue for the government, employment opportunities and community development. Its employees told us they appreciated relatively higher salaries and pensions, employee benefits and workplace camaraderie, exemplified below in a 'take-away' from our conversation with Bingwen, a trainee machine operator in his mid-20s:

Bingwen: I'm working at the Hongta Tobacco Factory. I'm a machine technician. Now I'm a trainee ... When I'm a permanent employee, my yearly salary will be 150,000 yuan ... I like the high salary and [the fact that] both of my parents worked here in the factory. I was already acquainted with many of my colleagues, so it's like going back home ... Cigarettes are free and unlimited for us in the factory; but we've limited break times, so we can't consume too much-about 10 cigarettes a day.

Smoking was permitted in the factory, and cigarettes were freely available, contributing to Bingwen's daily cigarette consumption (and, presumably, to its continuation rather than cessation).

Despite living in an industrial city, the residents we met had positive views about its air quality, environment and relaxed way of life. Yuxi was compared favourably to other cities such as Beijing with its notorious traffic congestion and smog. There was a consensus that living in Yuxi was conducive to good health. We heard only complaints about the high cost of living, which was attributed to the Hongta Group's relatively higher salaries; we too noticed that the prices of some food and daily necessities were slightly higher than in Kunming, the provincial capital (in China, living costs are usually highest in provincial capital cities due to relatively higher incomes and better facilities such as prestigious schools and hospitals).

Next, we took a taxi to the Hongta Industrial Tourism Park, a national tourist attraction and industrial area in the east of the city. Built in 2006 to satisfy an increasing appetite for domestic tourism, the park connects the tobacco industry to the city's heritage, and has created a narrative that tobacco is something worth commemorating. This was apparent as soon as we reached the park's main entrance where we were greeted by eight gold-coloured cigarette-like pillars, one of the tobacco-themed displays in the park and in the city (Figure 1).

Located opposite the Hongta Group, the display articulates more about Yuxi than might at first be apparent. The giant cigarettes that towered over our heads mark the eminence and power of cigarettes and of the tobacco industry, showcasing what 


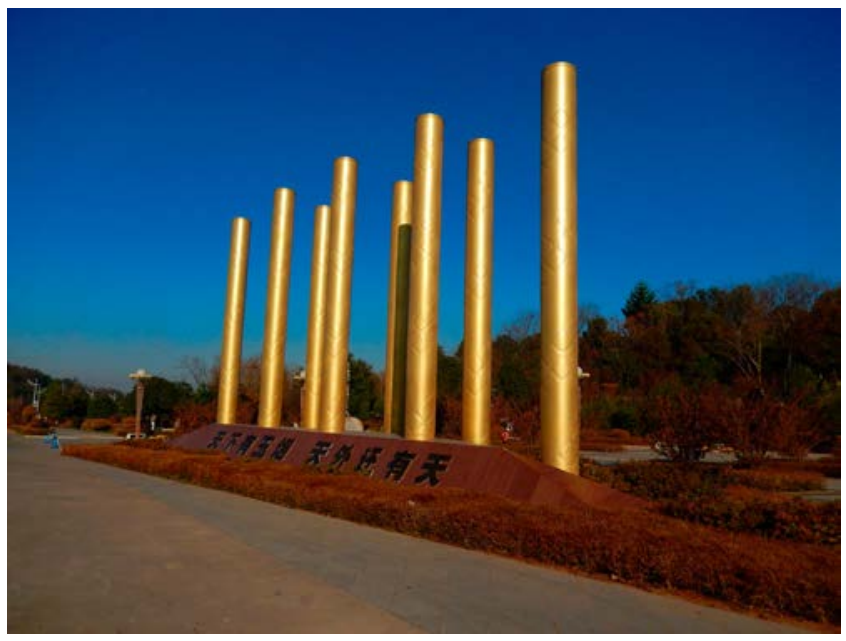

Figure 1 Eight Gold-coloured Cigarette-shaped Pillars at the Entrance of the Hongta Industrial Tourism Park, Opposite the Hongta Group.

Yuxi is all about. Gold-an auspicious colour in China-portrays the city's prosperity as built on cigarette production. The motto on the sculpture (天下有玉烟, 天外还有 天) implies that the cigarettes produced locally are highly reputed and can lead to further success-a message of inspiration for the park's visitors. Lin Li and Hua-Hie Yong (2009) reported similar examples of slogans and poems in tobacco advertising that they saw on the street in Kunming, connecting tobacco consumption and products to personal positive attributes and future possibilities (for example, happiness, luck), illustrating the ingenuity of tobacco promotion by local tobacco companies.

\section{Climbing Hongta Mountain}

We were out of breath as we climbed Hongta Mountain (红塔山, or its romanised form 'Hongtashan', literally meaning 'Red Pagoda Mountain'). At the top is a panoramic view of the Hongta Group's headquarters and main production facility, the heart of the 'city of tobacco' (Figure 2). We and the other visitors captured it photographically and shared the moment on social media. Remarkably, a strong and pleasant tobacco smell permeated the air.

The Red Pagoda rests on top of the mountain (Figure 3). Although originally white and known as the White Pagoda when built in 1839, during the Cultural Revolution it was repainted red-the emblematic colour of communism. Like the Tiananmen Square in Beijing, or the Bund in Shanghai, the Red Pagoda is an iconic symbol in Yuxi.

The Hongta Group is named after the famous landmark-as is its award-winning and most popular cigarette brand, Hongtashan. The Red Pagoda is also depicted pictorially on its company logo, and, therefore, on its cigarette packaging, retail tobacco store signage, advertisements and sponsorship of cultural and sports events. Thus, 


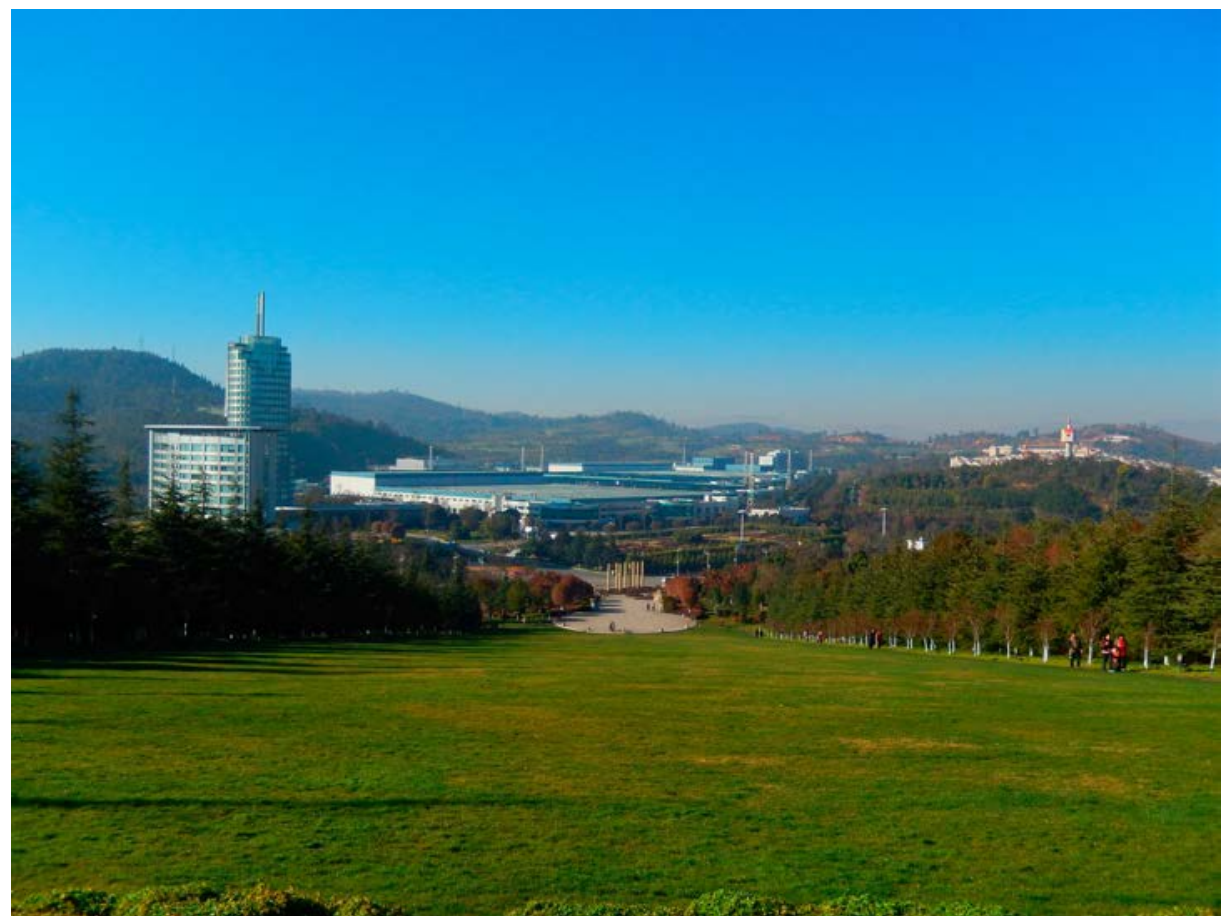

Figure 2 A View from Hongta Mountain Top: The Hongta Group's Headquarters.

the Red Pagoda and the Red Pagoda Mountain are recognisable throughout Chinaand also globally in the Hongta Group's exports. Many cigarette brands in China are associated with place, a branding strategy that began in the newly founded People's Republic after 1949 (Kohrman et al. 2018) (Figure 4).

The conflation of Yuxi, the Red Pagoda, and the Hongta Group and its tobacco products also featured prominently in our discussions. Eighteen-year-old Aiguo regarded these symbols as one and the same and as fundamental constructs in his definition of 'who I am' and 'where I am from'. Aiguo described himself and his ties to Yuxi using the tobacco industry:

Aiguo: I smoke Hongtashan ... because I am a 'Hongtashan person' ... I think they [Hongtashan and Yuxi] have the same meaning ... I like the Hongtashan brand. I smoke it, so I am a Hongtashan person ... for me, a Yuxi person is a Hongtashan person. The same meaning ... Many people in Shanghai didn't know Yuxi until I mentioned 'Yuxi tobacco'3... I was introducing myself like 'I am from the "Yuxi" that produces "Yuxi tobacco"', and then they just realised 'Oh, Yuxi is also a place name!'.

The demonym 'Hongtashan person' reveals the personal significance of the tobacco industry in Aiguo's sense of self and sense of place. Hongtashan is internalised 


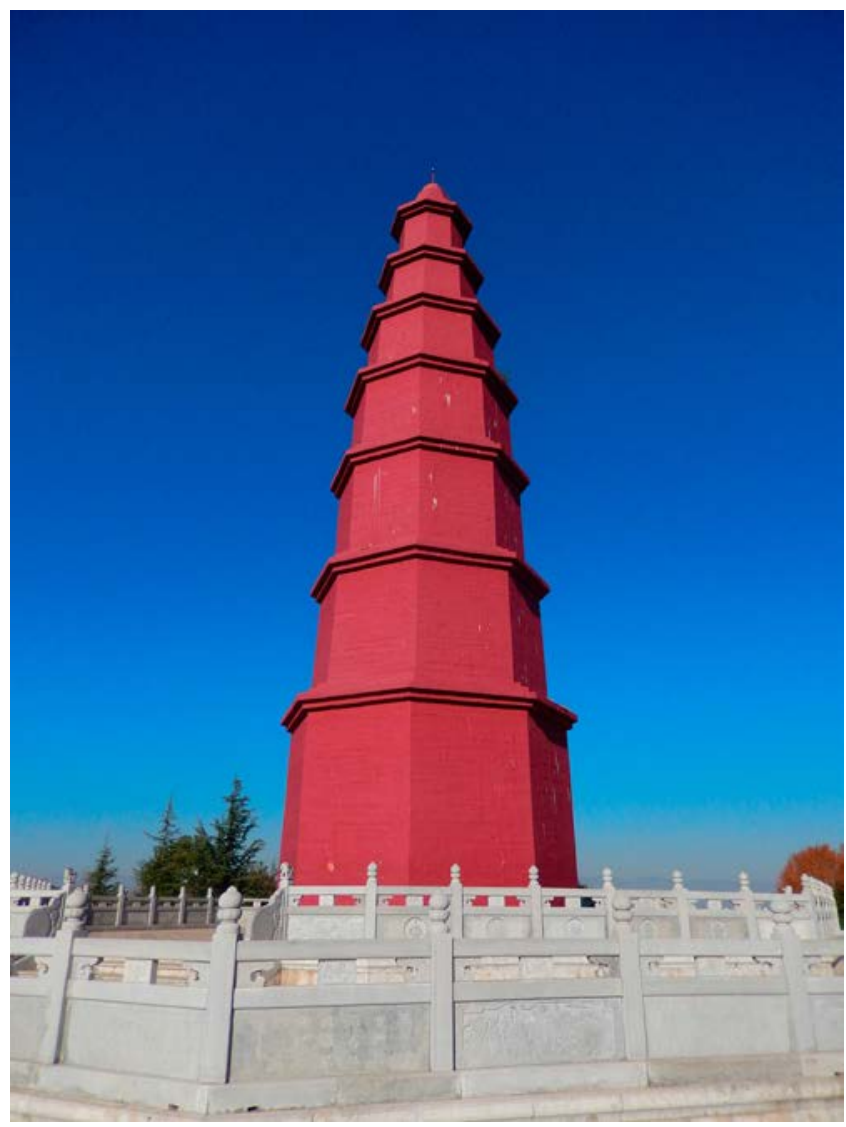

Figure 3 The Red Pagoda on Hongta Mountain.

cognitively in his consciousness and self-understanding, and externalised in his social self as a conversation starter when greeting and meeting strangers. In China, gifting and sharing cigarettes and lighters is common in greetings among people who smoke and in smoking socially (Rich and Xiao 2012). Clearly, the messages and symbols in cigarette branding and packaging are integral to the ways Aiguo thought and talked about himself, a psyche inextricably tied to the Hongta Group.

These tobacco-related symbols moor a common awareness of similarity and solidarity among people who smoke. Bingwen likened Hongtashan cigarettes to Yuxi's speciality, and his phrase 'hotpot in Sichuan' puts the brand on par with a wellknown Chinese cooking style characteristic of place:

Bingwen: It's a local brand, and I feel it's close to me. It feels like you're having something from home ... maybe some small places [outside Yunnan] don't have it, but in major cities you can always find it ... It's like hotpot in Sichuan. They [Sichuan people] are proud of it; and we are proud of our tobacco ... When I was in Xiamen and I smoked Hongtashan, it felt like I had 


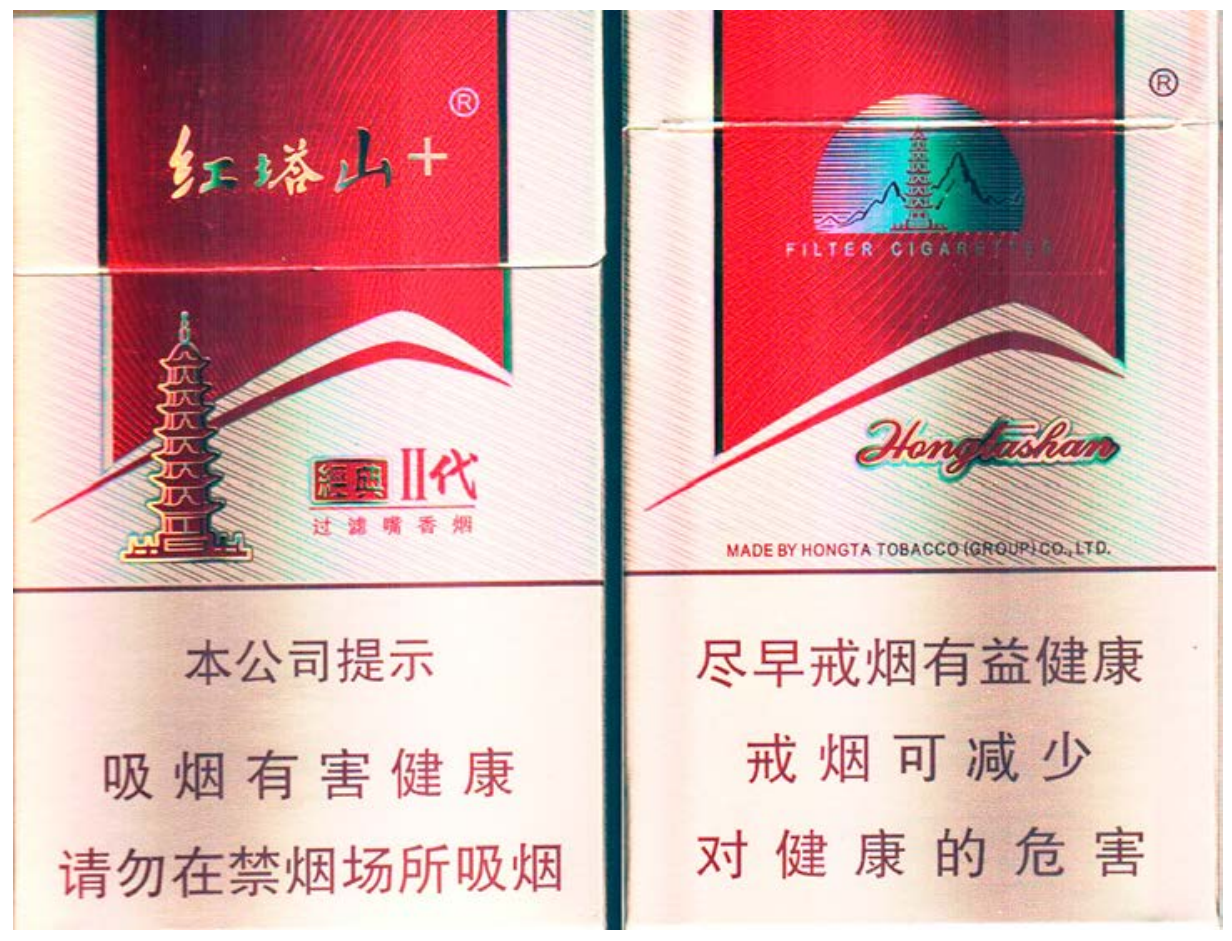

Figure 4 An Example of a Hongtashan Cigarette Packet Displaying the Red Pagoda and 'Hongtashan' in Chinese Calligraphy.

something from home... In the 1980s, Hongtashan already had national fame. People then often wore a white shirt with a Hongtashan packet in their shirt pocket. It was a way of showing off.

Bingwen felt connected to the city when he smoked Hongtashan cigarettes outside Yuxi. Fengge, a college student and part-time employee who we met at a McDonald's restaurant, became more aware of his sense of native place when he travelled to other provinces and mentioned Hongtashan cigarettes:

Fengge: Yuxi is my hometown. You don't feel so when you are here, but when you go to other provinces you realise it ... Not many people know Yuxi, but when you say Hongtashan, people may know it ... It's my hometown. I'm proud of it. People know the big brand name: Hongtashan. So, it's a famous place after the brand name. I feel I'm from here, and the famous cigarette is also from here. When people connect those things together, I feel great... as the tobacco industry is in the world's top 500 industries.

They were immensely proud of the tobacco industry in Yuxi. According to Bingwen, the Hongtashan brand was a fashion icon in Yuxi in the 1980s because of its national fame. Similarly, Fengge emphasised Hongtashan's reputation in China and felt happy 
when people associated it with his hometown. Indeed, Hongtashan is one of the most famous brands in the country (Li 2012). Fengge's comments 'so, it's a famous place after the brand name' and 'the tobacco industry is in the world's top 500 industries' ground his pride in the city on the economic might of the Hongta Group, which is regarded nationally as a flagship company of China's industry (Li 2012). These feelings form a sense of emotional satisfaction and security in place, a celebration of their identity as a native of Yuxi and as a person who smokes. Thus, the Hongta Group delivers satisfaction through its product's status and reputation and not only through its perceived quality.

Aiguo, Bingwen, and Fengge and others we met in Yuxi wore this place-based pride as a 'badge of honour' when befriending people, an image that was presumably respected by others too. It seems paradoxical that a place with an industry causing damaging health effects and even millions of deaths is revered as such. However, in China, unlike in Western countries, the tobacco industry is respected as a foundation of the economy, and smoking is much more socially acceptable (Hu 2013). Also, our interviewees fashioned a different take on smoking and health, one that emphasised its affective, sensory and social aspects rather than its biomedical or epidemiological interpretations fixed on morbidity and mortality (Tan 2013).

Importantly, these meanings of cigarettes and of smoking are at odds with the key assumptions of the tobacco control framework of our essay, namely that smoking is an action that is purely harmful to health and subject to stigmatisation and punishment (Robinson and Holdsworth 2013). Our emphasis on the causal significance of the tobacco industry in promoting a harmful practice should not be at the expense of people's own interpretations of their smoking and its situatedness in the contexts of their lives. In our data, comments about the benefits of smoking in everyday life far outnumber comments about its health consequences or other drawbacks such as nicotine addiction. The economic health of the city and of the country are prioritised over concerns about personal health. Our interviewees generally reported a low awareness of the health consequences of smoking, like many people we have interviewed in Yunnan over the past decade (for example, Davey and Zhao 2020). As Rebecca Haines-Saah $(2013,138)$ noted in her reflection on being a former smoker and a tobacco researcher,

Like the many adolescents and young adults I have interviewed since becoming a tobacco researcher, at the time I was certainly aware of, but had very little-perhaps almost no-concern about the health effects of smoking, with the immediate benefits it provided for me in my everyday life far outnumbering thoughts about the potential for future health harms.

The drawbacks of smoking have been played up in research literature on tobacco use in China, while its benefits have been glazed over, in line with its problematisation in public health. A critical anthropology of smoking reveals that the framing of smoking as a health hazard and as a deviant practice can fail to capture the cultural complexity of smoking (Dennis 2016), as suggested by our data. 
The Red Pagoda's symbolism has further significance. The colour red (Hongta) is steeped in political and national significance since it denotes the victory of the Chinese Communist Party and the establishment of the People's Republic in 1949. The Hongtashan brand was launched in 1959 to commemorate the 10th anniversary of the proclamation of the State, powerfully linking Hongtashan, the Hongta Group, and the 'city of tobacco' to official narratives of revolution, heroic victory and liberation. When a person lights a Hongtashan cigarette, or considers themselves as a 'Hongtashan person', it means much more than simply smoking or pride in the city and its tobacco company; it also acts as a chief symbolic expression of national loyalty and patriotism. Indeed, political propaganda in cigarette branding has a long history in China, and dates back to the beginning of the twentieth century and the brand development of the earliest manufactured cigarettes (Benedict 2011; Kohrman et al. 2018).

\section{Strolling Around the Tobacco Museum}

It took us a couple of minutes to walk to the Tobacco Museum, also situated on Hongta Mountain. Its name alone made it immediately apparent that we were at another significant site of identity construction. As we approached the museum grounds, we saw visitors taking photos of life-sized statues of tobacco farmers planting, harvesting and transporting tobacco leaf (Figure 5).

The statues are symbolic in every detail. The old-fashioned farming equipment is a throwback to previous times, making tobacco production in Yuxi historically significant-important for the city's identity (Cheshmehzangi 2020; Lynch 1960). Since China has a large rural population, including 22 million tobacco farmers (Li 2012), the museum exhibit conveys unity with 'the people', acting as a reminder that many livelihoods depend on the tobacco industry. Political connotations can be drawn as the peasantry has been a major force in the establishment of the State and its present-day prosperity, again linking the tobacco industry to the nationstate. The large tobacco seedlings in the exhibit signify the high quality of tobacco produced locally by the Hongta Group in fertile land and favourable climatic conditions; these seedlings are described metaphorically as 'golden tobacco fields' in the exhibit label (golden or gold is often used in Chinese to describe a bumper crop).

Upon entering the museum, we saw photo exhibits on the walls that show Mao Zedong and Deng Xiaoping smoking and smiling, an official endorsement of tobacco use at the highest level, and a coupling of the government and the tobacco industry as one and the same (Figure 6).

As well as glamourising tobacco with grandiose figures revered nationally, the exhibit entwines the tobacco industry and the 'city of tobacco' within national identity narratives of revolutionary history and economic and societal transformation. Mao Zedong is remembered in China as the founder and leader of the People's Republic in 1949, and Deng Xiaoping is regarded as the architect of the country's economic reforms and the opening up to the outside world since the late 1970s. Tobacco consumption is represented in the museum as central to these 'successes'. 

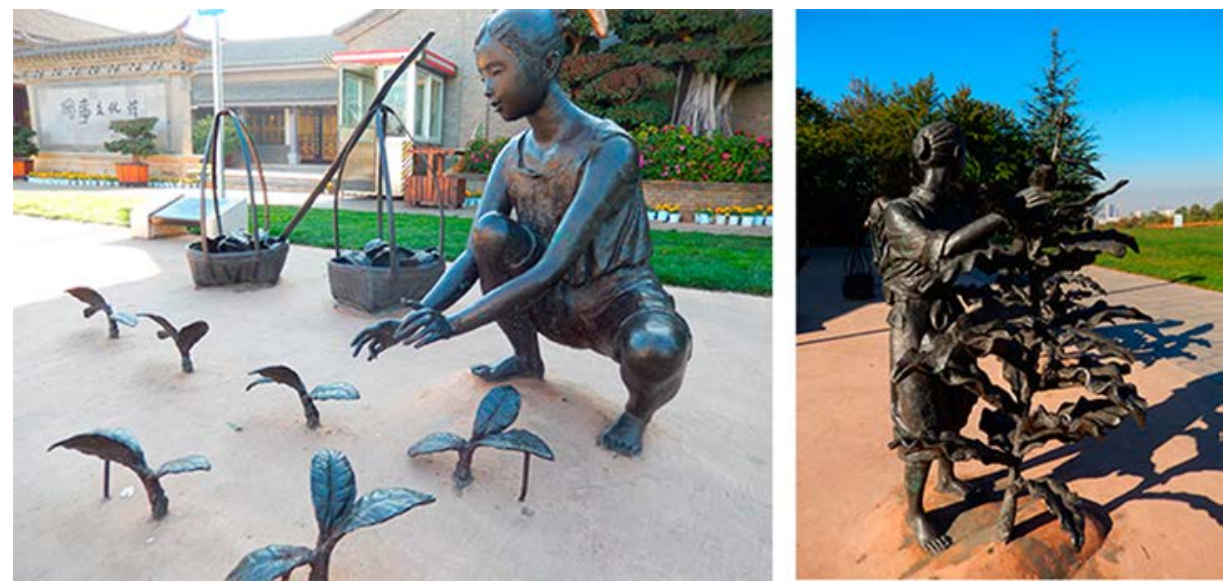

Figure 5 Life-sized Statues of Tobacco Farmers Outside the Tobacco Museum.

The photos of the leaders alongside each other imply continuity and durability in tobacco production and consumption through recent history, in line with the official rhetoric of State continuity, despite substantial disruption and transformation in both the Mao-era and the Deng-era.

The exhibit also reflects popular imagination in China of political leaders in the past as hard-working smokers, relating smoking to work ethic and personal and national success. It might also encourage smoking, as there is a long tradition of perfection through emulating Chinese political leaders and propaganda (Davey and Zhao 2020).

Next in the museum we saw photos of twentieth century celebrities posing while smoking or holding cigarettes, such as Albert Einstein, Alfred Hitchcock, Charlie Chaplin, Fidel Castro, Sigmund Freud and Winston Churchill. Here smoking is glamourised through its association with eminent actors, intellectuals, novelists, politicians, and scientists - the upper echelons of global society. They, too, are well known in China as fervent smokers, and the photos correspond to stereotypes of people who smoke as being in positions characterised by authority, privilege, power, success and wisdom (Davey and Zhao 2012), even though recent reductions in tobacco use in high-income countries have mostly occurred among the socially advantaged (Figure 7).

Almost all of the photos in the museum display men, consistent with the higher prevalence of smoking in men and its connotations of masculinity. The single photo of a Chinese ethnic minority person links the exhibit to Yuxi, as a sizeable proportion of its population are minorities. The only woman in the exhibit is also an ethnic minority, denigrating ethnic minority and women smokers as exotic and out of the ordinary, again reproducing stereotypes in society about people who smoke.

We strolled around the museum exhibits about past and present tobacco production and consumption-the Hongta Group's cigarette brands and packaging, 


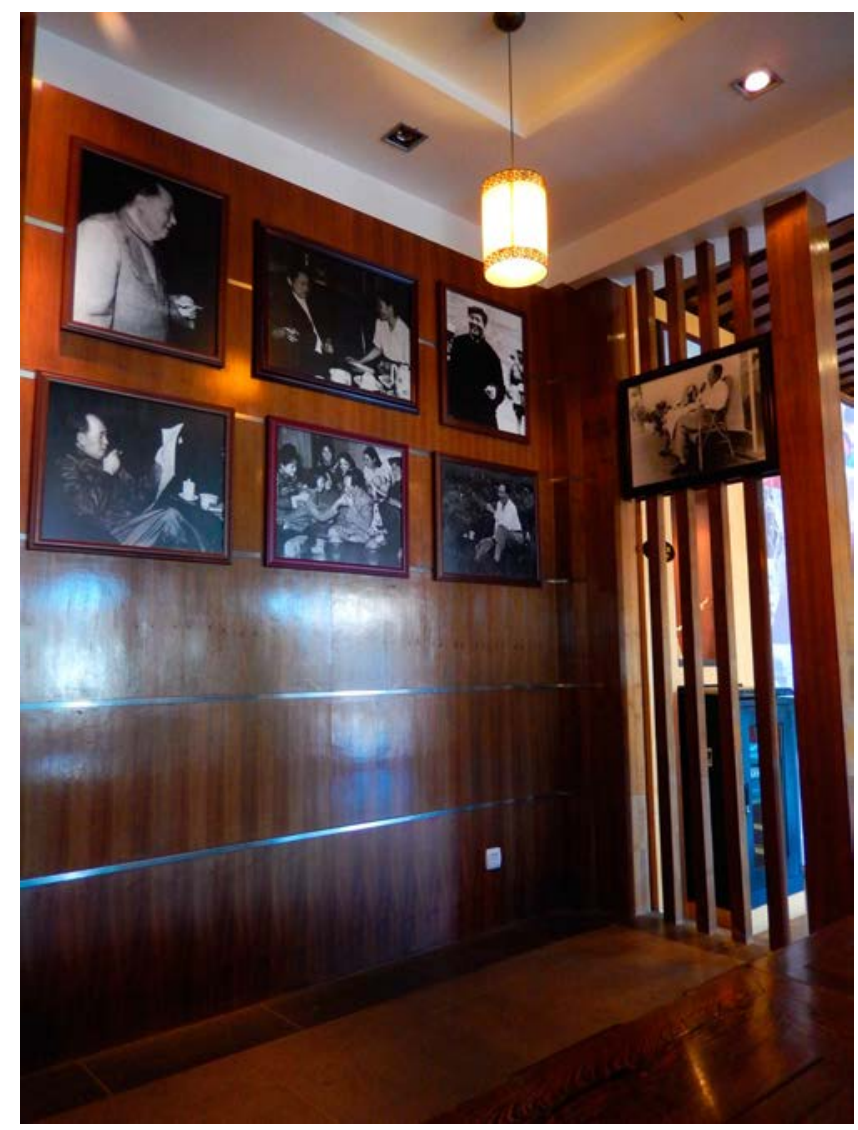

Figure 6 Photos in the Tobacco Museum of Smoking by Chinese Political Leaders.

and smoking paraphernalia such as cigarette cases, tobacco pipes, lighters and snuff bottles-which act to construct a city's history or a city's tradition based on tobacco.

We did not see any negative information about tobacco in the museum (for example, health dangers, quality of life of tobacco farmers), and there was no advice about quitting smoking. Photos of the current top Chinese leadership were also nowhere to be seen, perhaps because they do not smoke cigarettes or because they rarely do so in public. Ironically, cigarettes were sold in the museum, adding to its purpose (at least in our minds) of tobacco advertising and promotion. Similarly, Teh-Wei Hu (2013) regarded the China Tobacco Museum in Shanghai as an example of the efforts of the tobacco industry in its outreach activities to build public support and respectability. The funder of the museum and of the park in Yuxi is not clearly stated. Anecdotal records in official Yuxi Yearbooks suggest hidden links with the Hongta Group and the local government. For example, in late 2009 the two millionth box of Hongtashan cigarettes produced by the company became part of the museum's collection after a ceremony. Official local government documentation 


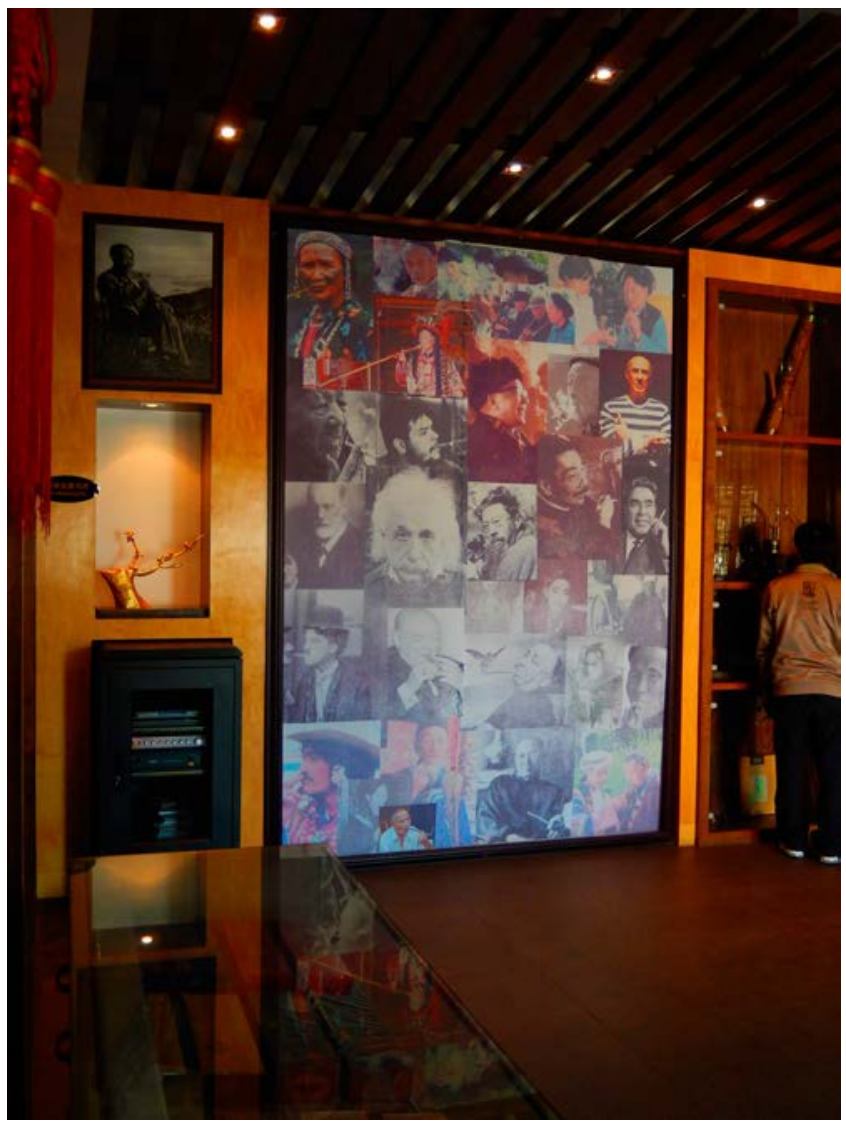

Figure 7 Photos in the Tobacco Museum of Smoking by International Celebrities and an Ethnic Minority Woman.

refers to the museum as the 'Hongta Tobacco Museum' (Hongta District Shizhi Bangongshi 2010, 234). In May 2012, the museum and the park were included in the itinerary of Cambodian senior officials visiting Yuxi (Yuxishi Difangzhi Bangongshi 2013).

Judging from several dialects that we overheard spoken by Chinese tourists in the museum and in the park, these messages were reaching diverse audiences from across the country. Fifteen reviews from museum visitors posted on Ctrip.com, an online travel agency, indicate they enjoyed the museum and regarded it as educational. However, while this might be true for domestic visitors, we feel that the museum severely undermines the international image of the city and of the country, and is at odds with China's projected image as a global and responsible 'power'. Located alongside Yuxi Normal University, it is likely that young people will be attracted to the park. Unfortunately, this audience is particularly susceptible to smoking experimentation and initiation, and vulnerable to the tobacco industry's efforts to 
recruit new smokers by making smoking look accessible, appealing and socially acceptable.

\section{Exploring Downtown and Beyond}

We continued our journey around Hongta District, and we learnt about the influence of the tobacco industry on smoking norms and practices. Most of the people we interviewed smoked Hongtashan cigarettes, a near monopolistic preference that was described as a norm and a custom among people who smoked in Yuxi, and also a key criterion of their identification in common. As we stopped by a pub for refreshments, Liang, a young bartender, told us why he smoked that brand:

Liang: I usually smoke Classic 100, a series by Hongtashan. I smoke it because it's a local brand. Sometimes I also try Honghe. But Hongtashan is still my favourite. Smoking a local brand is a habit here ... I tried others, but I still prefer Hongtashan. Maybe it's their taste and intensity ... Local people should smoke local cigarettes ... My friends also smoke this brand, and they offer me it. So, it gradually became a habit ... It's something belonging to Yuxi. I feel a bond, but don't know how to describe it.

Liang felt obliged to smoke cigarettes produced locally and to conform with others. He also described a taste preference for Hongtashan cigarettes, and he felt accustomed to them. Similar reasons were given by Qingshan, the middle-aged owner of the restaurant where we stopped for lunch:

Qingshan: I smoke Hongtashan. It's a local brand. I started smoking this brand. It's like a habit... I tried Guangdong tobacco, Jiangxi tobacco, and other tobacco products from many other places. I don't quite like them ... the taste is not my type ... We can't easily access other brands... As far as I know, most Yuxi people smoke Yuxi tobacco.

These excerpts from Liang and Qingshan uncover the privileging of the Hongtashan brand in claims of locality and superior taste, and in taste aversion for cigarettes produced in other places. They resemble tobacco advertising in Yunnan, which promotes the unique flavours of specific brands (Li and Yong 2009). These quotes also suggest that Liang's and Qingshan's cigarette brand preference and loyalty was established when they first tried cigarettes (DiFranza et al. 1994). Hongtashan is likely to be the first brand encountered in Yuxi by a person new to smoking, thus priming a brand loyalty that is perpetuated in social smoking norms. Indeed, Qingshan said cigarette brands produced in other places were unavailable in Yuxi. Protectionism of local tobacco markets practised by city and provincial governments to increase locally made cigarette production and consumption, and, therefore, government income, is well documented ( $\mathrm{Li}$ 2012). Another reason for limited access to brands could be the unviability of a business selling tobacco made in other provinces, as suggested below in Aiguo's quote. 
Exceptions to Hongtashan's domination do exist, however. A couple of our interviewees consumed tobacco products from other places in Yunnan such as in Kunming and Mile, although they encountered social pressure to smoke Hongtashan cigarettes. Some people did not smoke Hongtashan cigarettes when living outside Yunnan because of unavailability or because of concerns about counterfeits.

Cigarette smokers were categorised by our interviewees into distinct groups or identities according to the geographical location of cigarette brand production; for example, in Qingshan's descriptor 'Guangdong tobacco, Jiangxi tobacco'. Some people told us they could identify a person's place of origin according to the brand of their cigarettes: 'The main difference is brand choice. I smoke Hongtashan. Hong Kong smokers like Marlboro. Nanjing smokers like Huanghelou. Yunnan smokers like Honghe and Yuxi' [Tengfei];

Shanghai people tend to smoke Zhonghua. Fujian people tend to smoke Qipilang ... Kunming people like to smoke Yunyan. So, if I see some people smoking Yunyan on the street, it is very likely they are from Kunming; and, in Kunming, if you see someone smoking Yuxi tobacco, you can also guess where that person is from. [Bingwen]

Thus, in the processes of social categorisation, cigarettes and the people who smoke them are identified with place. This also pertains to outside China, for example Marlboro means 'foreign'. And it is reflected in the practice of embossing specific cities or provinces on cigarette packets.

The identification of a cigarette brand with a certain place was considered centrally important by smokers in relation to their peers. Aiguo gave us an example of its importance in smoking and gifting cigarettes with his colleagues in a factory in Shanghai:

Aiguo: I was in a factory with people from different provinces. They smoked tobacco produced in their own provinces ... I had some colleagues from Yuxi, and we smoked Yuxi brands, and we occasionally tried other brands ... It was a pleasant feeling. Every time I smoked, I showed others that the brand name was from my hometown ... We offered our cigarettes to others and nobody rejected them ... but in Yuxi, if you run a tobacco shop with only Hunan tobacco, for example, it wouldn't work out. If the shop has all foreign brands, maybe people would like to try... I know there is a shop here [in Yuxi] selling Korean tobacco. It has good business. It's about novelty. People see the writing on the package in a foreign language ... it is just curiosity.

The factory workers from Yuxi smoked the Hongta Group's cigarettes, and they gifted samples to their colleagues from different provinces. Similarly, their colleagues smoked and gifted their own local brands. In this way, everyone presents place-based identities to peers through cigarette branding. Smoking and gifting cigarettes to craft specific impressions and images in China is well documented (Davey and Zhao 2020; Rich and Xiao 2012). As Davey and Zhao $(2020,17)$ remind us, 'Like an actor wearing an elegant dress or tattered rags, cigarette brands are analogous to costumes in a play 
by crafting specific impressions and images ... and enabling the audience to size up the smoker and respond accordingly'.

Thus, we found that the meanings of place associated with cigarette brands make them especially appealing to those people seeking identity and acceptance, and that once a cigarette brand is attributed a certain status and has been integrated within one's sense of self and one's sense of belonging, its meaning and popularity are further reinforced through identity work, conspicuous consumption and smoking norm compliance. Furthermore, these marketing practices of the tobacco industry have become socialised and routinised in smoking, which inevitably maintains and supports both smoking and the products of local tobacco industries. Our findings lay bare the tenacious hold of the Hongta Group on people who smoke in Yuxi, as they remain loyal to the company throughout their smoking careers-even when they work in other cities and provinces. Indeed, Ding and Hovell (2012) go so far as arguing cigarette gifting in China to be engineered by the tobacco industry to build brand identity and increase cigarette consumption, as seen for example in the creation of certain meanings of specific brands and of cigarette gift boxes designed for auspicious festivals.

Yet our findings also suggest that the direction in which identity and brand consumption run might be recursive or even that both identity and brand choice are arrived at simultaneously; for instance, Aiguo's comment, 'Local [good] people should smoke local cigarettes [X local brand]', implies that the pre-existing quest to define one's identity can be fulfilled through adoption of a local brand, and thus brand preference might be determinative of marketing practices which function to reinforce identity. Further research is needed on identity and marketing in relation to the apparent behavioural and constitutive upshots of smoking norms.

As we reflect on these meanings of place in smoking, it is apparent that the public health and tobacco control framing of our study has limitations. People who smoke are typically portrayed in the academic literature as trapped in nicotine dependency and ignorance about the health consequences of smoking, a characterisation of passivity and mindlessness that can be remedied by rational health professionals and their interventions and education about smoking and health (Dennis 2016). In this essay, we have documented complex identity and social performances and rich contextual and social factors as alternative reasons for explaining people's smoking based on agency and consciousness and social benefits (Dennis 2013, 2016; Hunt and Barker 2001). Research about why people use tobacco and on its social and economic aspects beyond simple and dissatisfying explanations such as addiction (Black 1984; Dennis 2016; Levi-Strauss 1973), remains important.

Delong, a baker in his mid-20s, thought the omnipresence of tobacco symbolism in the city might have prompted his uptake of smoking, and he connected his pride in smoking to his pride in the tobacco factory in Yuxi. His friend, Guang, a young marketing professional, said he began smoking because he was curious about the smell of 
tobacco from the tobacco factory-we also noticed the smell of tobacco production in some areas of the city, as we travelled around:

Delong: I'm very proud. The biggest tobacco factory in China is here. Maybe I'm proud of being a smoker ... Living in Yuxi, I'm surrounded by everything about the tobacco factory. Maybe my smoking is influenced by it ... [Tobacco is] very important for me... It's the largest industry, and helps the economy. It also increases the popularity of the city.

Guang: I partly agree with my friend's idea. I started smoking as I was curious about the nice tobacco smell. When you are close to the factory, you can pick up a strong sense of the odour.

Smoking is perceived as more socially acceptable and popular in Yuxi than in other cities. Bingwen described tobacco use as a 'culture' in Yuxi, a reference to its normality and common practice, and likened it to a popular tradition or to a national dish:

Bingwen: In this city, smoking is a culture. People don't think you are bad if you smoke. I think people in Yuxi have more positive attitudes towards smoking ... Smoking is not a problem, but in Xiamen I'm mindful of the antismoking logo or other signs. Normally you can smoke anywhere in Yuxi ... I remember when I was in Xiamen the whole place was anti-smoking and I felt uncomfortable ... like British people having fish and chips, our culture here is having tobacco.

His acquaintance, Xiao, also thought it was easier to smoke in public in Yuxi as it was more acceptable and normal and because people who smoke were judged less negatively than in other places. Previous studies in other countries have found stronger pro-smoking attitudes in places more economically reliant on the tobacco industry (Ross and Taylor 1998). It has been suggested that there is little incentive for local governments in areas of tobacco production to enforce tobacco control initiatives, as doing so would challenge their revenue (Yang et al. 2015). These findings suggest that the regional differences in commitment to tobacco control that exist in the US in relation to economic reliance on tobacco manufacturing (Morley and Pratte 2013) might also exist in China.

These excerpts from the interview data also remind us that the focus in academic literature on the denormalisation of smoking, and on anti-smoking legislation, policies, and practices, mainly refers to high-income countries such as Australia, Canada, the United Kingdom and the United States, and their specific social and political conditions. Although tobacco control efforts are advancing in China, for example with the impact of the World Health Organisation Framework Convention on Tobacco Control (FCTC), smoking is much more socially acceptable and permissible in public places than in other countries. And, as we have documented, our interviewees were immensely proud of their smoking and of the tobacco industry, in line with public attitudes in China, unlike in high-income countries. This raises the question whether a pre-existing reality such as 'smoking as a problem' generalises to China 
in similar ways to other countries, and whether research anchored on reducing people's consumption of cigarettes and on anti-tobacco industry messaging is culturally appropriate when exploring smoking practices in China. To this end, further research could be undertaken to compare the social construction of smoking as a problem in China to other countries in relation to historical, social and political processes.

\section{Reading Newspapers in an Internet Cafe: A City Identity in Flux}

Using Duxiu, an Internet search engine specialising in Chinese academic resources, we searched for the word 'Yuxi' in the titles of news reports published between 1946 and 2018 in People's Daily, the most popular national newspaper in China. Official media such as People's Daily are operated by the Central Committee of the Chinese Communist Party, and can be regarded as its viewpoints or intentions or official statements.

A total of 130 news reports were identified. Reports from the 1950s to the 1970s refer mainly to agricultural technologies and policies; several between 1958 and 1963 concern the achievements and benefits of tobacco production. Articles from the late 1970s to 2000 emphasise the financial and societal contributions of the tobacco industry. From 1995 onwards, there is a shift in news reporting, initially to culture and sports competitions and, after 2000, to the city's infrastructure. Tobacco is mentioned only once in an article (July 7, 2002) about the popularity of cigarettes manufactured by the Hongta Group.

To triangulate these findings, we also searched for articles about Yuxi in three other official newspapers: Guangming Daily (38 articles between 1998 and 2018 ${ }^{4}$ ), The People's Liberation Army Daily (15 articles, 2000-2018), and Economic Daily (6 articles, 2000-2018). None of the articles about Yuxi published in Guangming Daily cover news about tobacco directly, and most relate to cultural or sports events. Additionally, from 2008 onwards, the newspaper reported on improvements in the city's environment. Reports in The People's Liberation Army Daily are mainly military-related and not connected to tobacco. The articles published in Economic Daily concern macro-level economic issues related to the city; a single article in 2002 argues that alternatives should be sought to its economic dependence on the tobacco industry.

What can we make of this preliminary analysis? There have been temporal shifts in national media representations of Yuxi: agricultural development prior to the 1970s, the tobacco industry and its achievements from the mid-1970s to the mid-1990s, and culture, environment, and society from 2000 onwards. We argue that since 2000 there has been a 'culturing' or a 'polishing' of the city's identification with tobacco through news stories about culture, environment and society. The construction of the Hongta Industrial Tourism Park in 2006 and the emphasis on the city's culture and history based on tobacco as heritage are also in line with these changes. The State media surveyed can perhaps be regarded as an official endorsement or even as a driver of this identity change in public consciousness, which is regarded as crafted by the Chinese 
Communist Party given that most people and institutions in China stick to the party line. They also legitimate its internalisation and expression by local residents, as evidenced in our finding that the city's polished tobacco identity has fed back to inform people's perceptions of the city and of themselves (for example, 'Hongtashan person').

It is not clear if the changing media representations of Yuxi reflect a strategic use of the media by the government or by the tobacco industry to balance images of tobacco production in the city with images of cultural heritage and social and environmental responsibility. The Hongta Group, however, has invested large sums of money in 'technical development' including tourism construction, for example more than 1 billion RMB in 2005 (Xuan 2005).

\section{Our Departure: Some Final Thoughts}

We reflected on our findings during the return journey to Kunming. The first research objective was to explore the meanings and practices of smoking in Yuxi. Most people in the world have an awareness of the places where they were born, grew up, and live (in adulthood) (Lynch 1960; Relph 1976). In Yuxi, a person's sense of self and sense of place are affectively, cognitively and socially tied to tobacco and to the tobacco industry. Place in cigarette branding and in the tobacco industry is highly appealing to smokers, and internalised in their identities, and socially valued and emotively realised, explaining allegiance to certain brands such as how and why Hongtashan has achieved popularity-a different take on smoking to the biomedical obsession with morbidity and mortality.

The second research objective was to describe the tobacco-related symbols and structural forms in the city. Tobacco imagery is highly visible and abundant and nearly everywhere we looked: in physical architecture, toponymic terminology, landmarks, museum education, tourism sites, and tobacco product brand names, corporate logos and packaging. Thus, cues promoting smoking and its uptake, continuation and normalisation, specifically the Hongta Group's products, are ubiquitous. Even the very existence of a 'city of tobacco' glorifies tobacco production and consumption-an all-encompassing tobacco advertisement. Since tobacco advertising is somewhat restricted in China, these examples of tobacco symbolism illustrate creativity and resourcefulness in tobacco advertising and marketing as well as possible loopholes in existing bans. We argue for a complete ban of all tobacco-related symbolism in the environment (other than about the hazards of tobacco use or its cessation), and for architects, designers and urban planners to be involved in this endeavour. This needs to be addressed through skilful collaboration with national and local governments, as tobacco symbolism is coded with nationalist and patriotic narratives, conceivably by the State to conjure public support and by the tobacco industry to allure customers - a kind of State-authorised tobacco discourse in society. The conjoining of tobacco and of the nation-state in this symbolism is not entirely surprising, since the Chinese tobacco industry is a gigantic and powerful government monopoly and revenue generator, and Chinese Commuist Party leaders and provincial leaders have played critical roles in the Hongta Group's development (Li 2012). 
The third research objective was to examine representations of Yuxi in official national newspaper content from 1946 to 2018. It is not clear if the recent shift in newspaper reporting from tobacco production and its economic importance to the culture, society and environment in Yuxi is a strategic use of the media by the government or by the tobacco industry to present themselves more positively. However, the tobacco industry's efforts to create favourable public images through contributions to culture and society are well documented (Hu 2013; Yang et al. 2010).

Fourth, our interviewees alleged that living within the vicinity of the Hongta Group had contributed to their smoking initiation and consumption, pro-smoking attitudes and pride in smoking. The presence of the tobacco company might have also contributed to more relaxed tobacco control in Yuxi. These are potential explanations for the suggestion that smoking prevalence is highest in provinces with the highest rates of cigarette production (Yang et al. 2015).

Alarmingly, smoking and tobacco are enmeshed in the entire fabric of the city and of people's lives. Inspired by the work of Deleuze and Guattari (1987), we characterise this integrated and totalising phenomenon as 'rhizomatic smoking'. While we are not questioning the value of interventions designed to help people to quit smoking, it is clear that isolated and small-scale anti-smoking and tobacco control initiatives-such as identifying and targeting component parts or 'pathways' between health and place variables, or regarding smoking as an individual health behaviour driven by personal attitudes and knowledge and specific variables-will only scratch the surface of this rhizomatic beast, which probably explains why smoking cessation interventions in China hitherto have been unsuccessful. We call for 'rhizomatic tobacco control' as a new direction for thinking about the entirety of tobacco and its symbolism in society, and to subject tobacco control to critical scrutiny. As highlighted in our reflexivity throughout this essay, sole emphasis on smoking as a health hazard and as a deviant practice not only fails to capture its cultural complexity but could even do more harm than good, for example by not resonating with people's reasons for smoking, or by misleadingly characterising people who smoke as passive objects of control and intervention, or by uncritically assuming that people have stopped smoking as a result of anti-smoking campaigns (Bell and Dennis 2013; Dennis 2016; Haines-Saah 2013; Robinson and Holdsworth 2013). 'Rhizomatic tobacco control' should engage with growing calls for a critical anthropology of smoking to enable it to effectively meet the needs of people who smoke and to be relevant to their everyday contexts and realities.

Perhaps we can take solace in the fact that the social construction of tobacco, the tobacco industry, and the 'city of tobacco' is not universal or fixed, and alternative positions exist. Although our research was conducted within the framing of public health, we are not suggesting that only a commitment to tobacco control legitimises tobacco research. Also, the perpetual indeterminacy of identities means that our journey in Yuxi can be regarded only as a snapshot of cultural and social production in 2014 and 2015 when we conducted a small pilot study to explore ideas rather than make definitive conclusions. Clearly, understanding a complex city with a handful of 
selected data, which are multi-faceted and sometimes abstract, mitigates against making strong claims. Nor are we attempting to reify a city as an island or to fix its meanings and significance as natural and given, as we have been looking at identity as process rather than as a state of being. Finally, while our study found tobacco symbolism in Yuxi to be totalising, this is not to say that there really is a deliberately coherent and strategic whole or pattern or that Yuxi is culturally homogenous.

\section{Acknowledgements}

We gratefully acknowledge valuable feedback on our work from the TAPJA Editor and Executive Editor, and the journal's anonymous reviewers.

\section{Notes}

[1] It is important to note the polysemy of 'Hongta'. It is the romanisation of '红塔', literarily meaning 'Red Pagoda', and it is the place name of the Hongta Tobacco Company and of the Hongta District. We also use 'Red Pagoda' to describe the architecture in Yuxi.

[2] A sample of 22 men and 2 women aged 18-65 years. The larger portion of men reflected the higher prevalence of smoking in men in China.

[3] Aiguo referred to locally made cigarettes as 'Yuxi tobacco'. It should not be confused with a cigarette brand called 'Yuxi' which is produced by the Hongta Group.

[4] Unlike the online archive of People's Daily comprising all of its articles published since its conception in 1946, the other newspapers surveyed only had digitalised articles dating to about 2000.

\section{ORCID}

Gareth Davey (i) http://orcid.org/0000-0001-7237-2741

Xiang Zhao (D) http://orcid.org/0000-0003-1054-9462

\section{References}

Barnett, R., G. Moon, J. Pearce, L. Thompson, and L. Twigg. 2017. Smoking Geographies: Space, Place and Tobacco. Chichester, West Sussex: John Wiley \& Sons, Ltd.

Bell, K., and S. Dennis. 2013. "Editor's Introduction: Towards a Critical Anthropology of Smoking: Exploring the Consequences of Tobacco Control." Contemporary Drug Problems 40 (1): 3-20.

Benedict, C. 2011. Golden-silk Smoke: A History of Tobacco in China, 1550-2010. Berkeley, CA: University of California Press.

Black, P. 1984. “The Anthropology of Tobacco Use: Tobian Data and Theoretical Issues.” Journal of Anthropological Research 40 (4): 475-503.

Buttimer, A., and D. Seamon, eds. 1980. The Human Experience of Space and Place. London: Croom Helm.

Cheshmehzangi, A. 2020. Identity of Cities and City of Identities. Singapore: Springer Nature Singapore.

Davey, G., and X. Zhao. 2012. “A Real Man Smells of Tobacco Smoke-Chinese Youth's Interpretation of Smoking Imagery in Film." Social Science \& Medicine 74 (10): 1552-1559. 
Davey, G., and X. Zhao. 2020. “Turning Points to Becoming a Tobacco Smoker: Smoking Initiation and Identity Change among Chinese Youth." Symbolic Interaction 43 (2): 308-331.

Deleuze, G., and F. Guattari. 1987. A Thousand Plateaus: Capitalism and Schizophrenia. Translated by B. Massumi. Minneapolis, MN: University of Minnesota Press.

Dennis, S. 2013. "Golden Chocolate Olive Tobacco Packaging Meets the Smoker You Thought You Knew: The Rational Agent and New Cigarette Packaging Legislation in Australia." Contemporary Drug Problems 40 (1): 71-97.

Dennis, S. 2016. Smokefree: A Social, Moral and Political Atmosphere. London: Bloomsbury Academic.

DiFranza, J. R., J. J. Eddy, L. F. Brown, J. L. Ryan, and A. Bogojavlensky. 1994. "Tobacco Acquisition and Cigarette Brand Selection Among Youth.” Tobacco Control 3: 334-338.

Ding, D., and M. F. Hovell. 2012. "Cigarettes, Social Reinforcement, and Culture: A Commentary on 'Tobacco as a Social Currency: Cigarette Gifting and Sharing in China'." Nicotine \& Tobacco Research 14 (3): 255-257.

Erikson, E. H. 1950. Childhood and Society. New York: W. W. Norton \& Co.

Haines-Saah, R. J. 2013. "After the Smoke has Cleared: Reflections from a Former Smoker and Tobacco Researcher." Contemporary Drug Problems 40 (1): 129-153.

Hongta District Shizhi Bangongshi. 2010. Hongta Yearbook 2010. Luxi: Dehong minzu chubanshe. [in Chinese].

Hu, T.-W. 2013. "WHO Framework Convention on Tobacco Control in China: Barriers, Challenges and Recommendations." Global Health Promotion 20 (4): 13-22.

Hunt, G., and J. C. Barker. 2001. "Socio-cultural Anthropology and Alcohol and Drug Research: Towards a Unified Theory." Social Science \& Medicine 53: 165-188.

Kohrman, M., G. Quan, L. Wennan, and R. N. Proctor, eds. 2018. Poisonous Pandas: Chinese Cigarette Manufacturing in Critical Historical Perspectives. Stanford: Stanford University Press.

Levi-Strauss, C. 1973. From Honey to Ashes: Introduction to a Science of Mythology. New York: Harper and Row.

Li, C. 2012. The Political Mapping of China's Tobacco Industry and Anti-Smoking Campaign. Washington, DC: Brookings Institution.

Li, J., and D. Collins. 2017. "Smoking Environments in Transition: The Experiences of Recent Chinese Migrants to Canada." Health and Social Care in the Community 25 (1): 65-74.

Li, L., and H.-H. Yong. 2009. "Tobacco Advertising on the Street in Kunming, China." Tobacco Control 18 (1): 1-6.

Lynch, K. 1960. The Image of the City. Cambridge, MA: The MIT Press.

Mak, K. K. 2012. "Smoking Accessories for Cessation? Mixed Messages in Hong Kong." Tobacco Control 21: 366-367.

Morley, C. P., and M. A. Pratte. 2013. "State-level Tobacco Control and Adult Smoking Rate in the United States: An Ecological Analysis of Structural Factors." Journal of Public Health Management and Practice 19 (6): E20-E27.

Pearce, J., and P. Boyle. 2005. "Is the Urban Excess in Lung Cancer in Scotland Explained by Patterns of Smoking?” Social Science \& Medicine 60 (12): 2833-2843.

Pearce, J., R. Hiscock, G. Moon, and R. Barnett. 2009. “The Neighbourhood Effects of Geographical Access to Tobacco Retailers on Individual Smoking Behaviour." Journal of Epidemiology and Community Health 63 (10): 69-77.

Relph, E. 1976. Place and Placelessness. London: Pion Limited.

Rich, Z. C., and S. Xiao. 2012. "Tobacco as a Social Currency: Cigarette Gifting and Sharing in China." Nicotine \& Tobacco Research 14 (3): 258-263. 
Robinson, J., and C. Holdsworth. 2013. “They Don't Live in My House Every Day': How Understanding Lives Can Aid Understandings of Smoking." Contemporary Drug Problems 40 (1): 47-70.

Ross, N. A., and S. M. Taylor. 1998. "Geographical Variation in Attitudes Towards Smoking: Findings from the COMMIT Communities." Social Science \& Medicine 46 (6): 703-717.

Scheffels, J. 2008. "A Difference That Makes a Difference: Young Adult Smokers' Accounts of Cigarette Brands and Package Design.” Tobacco Control 17: 118-122.

Stead, M., S. MacAskill, A. M. MacKintosh, J. Reece, and D. Eadie. 2001. "It's as if You're Locked In': Qualitative Explanations for Area Effects on Smoking in Disadvantaged Communities.” Health \& Place 7 (4): 333-343.

Tan, Q. H. 2013. "Smoking Spaces as Enabling Spaces of Wellbeing." Health \& Place 24: 173-182.

Tuan, Y.-F. 1980. "Rootedness Versus Sense of Place." Landscape 24: 3-8.

Wang, J. 2012. State-market Interactions in China's Reform Era: Local State Competition and Global Market Building in the Tobacco Industry. Abingdon, Oxon: Routledge.

Xuan, Y. 2005. "Yuxi ‘shengtai lishi' zengqiang fazhan houjin." People's Daily, July 29. [in Chinese].

Yang, T., R. Barnett, I. R. H. Rockett, X. Y. Yang, D. Wu, W. Zheng, and L. Li. 2015. "The Impact of Regional Economic Reliance on the Tobacco Industry on Current Smoking in China." Health \& Place 33: 159-171.

Yang, Y., L. Li, H. H. Yong, R. Borland, X. Wu, Q. Li, C. Wu, and K. Foong. 2010. "Regional Differences in Awareness of Tobacco Advertising and Promotion in China: Findings from the ITC China Survey." Tobacco Control 19 (2): 117-124.

Yuxishi Difangzhi Bangongshi. 2013. Yuxi Yearbook 2012. Luxi: Dehong minzu chubanshe. [in Chinese].

Zhao, X., and G. Davey. 2015. "Contesting Modernity: Tobacco Use and Romanticism among Older Dai Farmers in Xishuangbanna, China.” Sociology of Health \& Illness 37: 1173-1190. 